July 1988

CEPREMAP

142. TUL IOTHEQUE

75013 PARis

Tél. : 4077 PARIS

$N^{\bullet} 8814$

ASYMPTOTIC PROPERTIES OF A LEONTIEF ECONOMY

\author{
Rose-Anne DANA ( $*$ ) \\ Mon ique FLORENZANO $(* *)$ \\ Cuong LE VAN $(* *)$ \\ Dominique LEVY $(* *)$
}

(*) Université de Paris VI

$(* *)$ C.N.R.S/C.E.P.R.E.M.A.P 
Résumé. Dans ce papier nous étudions les propriétés asymtotiques d'un équilibre d'une économie de Leontief où les agents ont des fonctions d'utilité séparables. Nous donnons une estimation des taux de croissance des quantités et des prix d'équilibre qui tendent vers zéro ou l'infini ou restent bornés inférieurement et supérieurement par des vecteurs strictement positifs. Dans le cas des préférences homogènes nous montrons que tout équilibre d'une économie de Leontief converge vers un équilibre qui croît à taux constant.

Mots Clé. Economie de Leontief, Equilibre général intertemporel, Prix de production, Préférences homogènes.

\section{ASYMTOTIC PROPERTIES OF A LEONTIEF ECONOMY}

Abstract. In this paper we discuss the asymtotic properties of equilibrium quantities of a Leontief economy when agents have additively separable utilities. We estimate the rate of growth of equilibrium quantities and prices that either tend to zero or infinity or remain bounded below and above by strictly positive vectors. In the case of homogeneous preferences we prove that the quantities of any equilibrium converge to those of an equilibriuin that grows at a constant rate.

Key words. Leontief Economy, Intertemporal General Equilibrium, Production Prices, Homogeneous Preferences.

J.E.L. $021,111,213$ 


\section{INTRODUCTION}

This paper attempts to link the theory of intertemporal general equilibrium and the neo-Ricardian theory of prices of production.

Prices of production have been introduced originally in the works of the classics, and later formalized by neo-Ricardian economists. Prices of production associate with a given wage rate a profit rate which is the same for every producer. In section five of its "Elements", entitled "The Theory of Capitalization and Credit", Walras defined equilibrium by the two following properties : i) all markets for commodities and factors clear and ii) a uniform rate of return is established for each entreprise. Actually, within a static framework of analysis, these two properties are not compatible. Prices of production in general differ from general equilibrium prices. For this reason, the definition of Walrasian equilibrium after Walras was restricted to the consideration of the first property. Prices of production were later introduced in the Von Neumann literature as prices sustaining paths of maximal balanced growth. Turnpike results were then discussed when the objective was to maximize terminal stocks (see Morishima [1961], McKenzie [1963]). This literature does not involve consumption.

The question of whether in an intertemporal setting equilibrium prices could lead to a uniform rate of profit was only adressed recently.

Hahn (1982) first attempted to draw a relationship between the two types of prices in the short run. He argued that prices of production are equilibrium prices, for a two period general equilibrium model with a Leontief technology, for well chosen values of the initial endowments. He concluded that prices of production correspond only to a particular case of general equilibrium prices and, therefore, are not very interesting from the point of view of economic theory. In a rejoinder to Hahn, Dumenil and Levy (1985) considered an infinite horizon model with a unique consumer, a fixed coefficient technology and an exogenous given real wage and claimed that prices supporting an optimal plan of consumption "could be chosen so as to converge to the prices of production. 
Dana et a1. [1987] carried the ir attempt further. They considered an intertemporal general equilibrium model with finitely many, infinitely lived consumers, and finitely many, infinitely lived firms. On the production side, the ir assumptions are those of a Leontief economy, in an intertemporal framework. There are as many firms as goods; at each period, each firm produces one good with constant returns to scale. For each good, a technology which is stationary over time relates substitutable inputs of the previous period to the production of one unit of output of the present period. On the consumption side, heterogeneous consumers have endowments of goods in the first period; the ir preferences for present and future consumption are represented by utility functions.

They obtained the following results : Under standard assumptions (but without assuming additively separable utilities), this intertemporal model has an equilibrium such that at each period the rate of maximum profit is uniform. The second result is in the spirit of Samuelson Solow's [1953] theorem. Let us consider prices of production as stationary prices which equalize the rate of maximum profit ; then the sequence of the undiscounted prices of the equilibrium defined above converges to vector of prices of production.

In this paper we discuss the asymptotic properties of equilibrium quantities when agents have additively separable utilities. Following Peleg and Ryder [1972], we characterize competitive equilibrium by intertemporally decentralised conditions. We then estimate the rate of growth of equilibrium quantities and prices that either tend to zero or to infinity or remain bounded be low and above by strictly positive rectors.

In the case of homogeneous preferences we show the existence of equilibria sustained by prices of production which grow at a constant rate. We then prove that the quantities of any equilibrium converge to those of an equilibrium that grows at a constant rate. This result can be compared to those obtained by Bewley [1982], Yano [1984], [1985], Coles [1985, 1986] but is based on a duality argument that uses the asymptotic properties of equilibrium prices. The paper is organised as follows. 
In section two we define the intertemporal model.

In section three we recall results obtained on existence of equilibria in our previous paper and characterize equilibria by means of decentralised conditions.

In section four, we study the asymptotic properties of the model. 


\section{Definition and properties of the intertemporal general equilibrium model}

Consider $m$ consumers, $n$ producers, $n$ goods produc ible by means of goods. Assume that the time horizon of the economy is infinite. Let $x_{t} \in R_{+}^{n}$ and $y_{t} \in R_{+}^{n}$ denote commodity bundles at time $t$ and let $p_{t}$ be a vector of prices at period $t$. Elements of the commodity space and price space of the economy will be denoted by $\underset{\sim}{x}=\left(x_{t}\right), \underset{\sim}{y}=\left(y_{t}\right)$ and $\underset{\sim}{p}=\left(p_{t}\right)$ and belong to $\left(R^{n}\right)^{\infty}$.

$\underset{\sim}{\sim} \cdot \underset{\sim}{x}=\sum_{t=0}^{\infty} p_{t} \cdot x_{t}$ will denote the present value of the commodity bundle path $\underset{\sim}{x}$ for the price sequence $\underset{\sim}{p}$.

We will use in $R^{n}$ and $\left(R^{n}\right)^{\infty}$ the following conventional notations : in $R^{n}, z^{\prime} \geq z \Leftrightarrow \forall k=1, \ldots, n, z_{k}^{\prime} \geq z_{k} ; z^{\prime}>z \Leftrightarrow z^{\prime} \geq z$ and $z^{\prime} \neq z$

$$
z^{\prime} \gg z \Leftrightarrow \forall k=1, \ldots, n, z_{k}^{\prime}>z_{k} \text {. }
$$

In $\left(R^{n}\right)^{\infty}$, if $\underset{\sim}{z^{\prime}}=\left(z_{t}^{\prime}\right)$ and $z=\left(z_{t}\right)$

$$
\begin{aligned}
& \left.z_{\sim}^{\prime} \geq z_{\sim} \Leftrightarrow \forall t \geq 0, z_{t}^{\prime} \geq z_{t} ; z_{\sim}^{\prime}\right\rangle \underset{\sim}{z} \Leftrightarrow \underset{\sim}{z} \geq z_{\sim}^{\prime} \text { and } z_{\sim}^{\prime} \neq z_{\sim}^{z} \\
& \text { Economy } \mathcal{C} \text { is described by the } 11 \text { st : } \\
& \mathcal{E}=\left(\left(\left(R_{+}^{n}\right)^{\infty}, U^{i}, S^{i}\right), A^{j}, i=1, \ldots, m ; j=1, \ldots, n\right)
\end{aligned}
$$

each term of which is defined below.

\section{Production}

There are $n$ producers and $n$ productble goods. At each period, producer $j$ produces, with constant returns to scale, an output $y^{j} \in R_{+}$of good $j$ from inputs of labour and goods available at the provious period. For all $j=1, \ldots, n$, let us denote by $A^{j}$ the set of inputs $\left(a^{j}\right)$, to produce one unit of good $j$.

Define :

$$
A=\prod_{j=1}^{n} A^{j}
$$


Note that $a=\left(a^{j}\right) \in A$ belongs to $R^{n^{2}}$ and can be identified to a $(n \times n)$-matrix.

We make on $A$ the following assumptions :

$1 \quad P_{1}-\forall j=1, \ldots, n, A^{j}$ is a non-empty, convex and closed subset of $R_{+}^{n}$

$P_{2}$ - All matrices in $A$ have the same strictly positive row

$P_{3}$ - For every proper and non empty subset $J$ of $\{1, \ldots, n\}$, there exist $k \in J$ and $j \& J$ such that inf $a_{k}^{j}>0$. $a_{\varepsilon A}^{j}$

$P_{2}$ assumes that one good is necessary for the production of all goods.

Assumption $P_{3}$ can be interpreted as a "non-splitablitity" condition for the production system. It means that for every non -trivial subgroup of producers, some good produced by a producer of this subgroup is a strongly necessary input (an input which cannot tend to 0 ) for the production of some producer of the complementary subgroup.

Let $\lambda(a)$ denote the dominant eigenvalue of matrix a.The following result Is proved in Dana et al. [1987].

Proposition 2.1 - Under assumptions $P_{1}-P_{3}$

1) $\lambda *=\inf \{\lambda(a), a \varepsilon A\}$ is strictly positive and there exists a* such that $\lambda *=\lambda(a *)$

2) There exists a unique $p * \gg 0$ such that $\lambda * p *=p * a *$ and $(p * a *)_{j}=\inf \left\{p * a^{j}, a^{j} \in A^{j}\right\}$ for every $j$.

We assume moreover

$P_{4}$ There exists a unique a* in $A$ such that $\lambda * p *=p * a *$

Bemark : $P_{4}$ is fulfilled if we assume that $A^{j}$ is strictly convex for every $j$. 


\section{Consumption}

There are $m$ consumers who own firms. Consumer $i$ has at time 0 an initial endowment $S^{i} \in R_{+}^{n}$. A consumption path for consumer $i$ is a sequence $\underline{x}^{i}=\left(x_{t}^{i}\right) \in\left(R_{t}^{n}\right)^{ \pm}$. Intertemporal preferences of consumers are assumed to be represented by a utility function $U^{i}:\left(R_{+}^{n}\right)^{\infty}+\bar{R}_{+}$and we make the following assumptions :

$$
\begin{aligned}
& c_{1}-\forall i=1, \ldots, m, \text { every point of }\left(R_{+}^{n}\right)^{\infty} \text { is a feasible consumption path. } \\
& c_{2}-\forall i=1, \ldots, m, S^{i}>0 \text { and } S=\sum_{i=1}^{m} S^{i} \gg 0 . \\
& c_{3}-\forall i=1, \ldots, m, U^{i}\left(x^{i}\right)=\sum_{t=0}^{\infty}\left(\delta^{i}\right)^{t} u^{i}\left(x_{t}^{i}\right) \text { where } u^{i}: R_{+}^{n} \rightarrow R_{+} \text {is }
\end{aligned}
$$

strictly increasing, strictly concave, continuous and satisfies $u^{i}(0)=0$.

$$
\begin{aligned}
& c_{4}-\forall i=1, \ldots, m, \exists \mu_{i} \in 10,1 c, \exists M_{i}>0, \exists m_{i}>0 \\
& m_{i}\|x\|^{\mu_{i}}<u^{i}(x)<M_{i}\|x\|^{\mu_{i}}, \forall x \in R_{+}^{n} \\
& c_{5}-\max _{i}\left(\delta^{i}\right)^{\frac{1}{\mu_{i}}}<\min (\lambda *, 1)
\end{aligned}
$$

\section{Attainable allocation paths}

An attainable allocation path is a triple

$$
\underset{\sim}{\sim} \underset{\sim}{y, a} \sim \prod_{i=1}^{m}\left(\left(R_{+}^{n}\right)^{\infty}\right) \times\left(R_{+}^{n}\right)^{\infty} \times A^{\infty}
$$


which satisfies the following relations :

(1) $\sum_{i=1}^{m} x_{0}^{i}+a_{1} y_{1} \leqq s$
(2) $\sum_{i=1}^{m} x_{t}^{i}+a_{t+1} y_{t+1} \leqq y_{t}, \forall t \geqq 1$

We will say that each of $\underset{\sim}{y}, \underset{\sim}{a} \underset{\sim}{x}$ is attainable if it is a part of an attainable allocation path.

Let $\hat{x}(s)$ be the set of all $x$ attainable with a total initial endowment equal to $s, \hat{x}^{i}(s)$ the set of all ${\underset{x}{i}}^{i}$ attainable with $s$ and $\hat{C}(s)=\left\{c \in\left(R_{+}^{n}\right)^{\infty} / c=\sum_{i=1}^{m} x_{i}, x \in \hat{X}(s)\right\}$.

Prooosition 2.2 - Under assumptions $P_{1}-P_{3}, C_{1}-C_{2}$

1) $\quad \forall s \in R_{+}^{n}, \hat{C}(s)=\left\{\underset{\sim}{c} \varepsilon\left(R_{+}^{n}\right)^{\infty} \mid c_{0}+\sum_{t=1}^{\infty}\left(\prod_{\tau=1}^{t} a_{\tau}\right) c_{t} \leqq s, \underset{\sim}{a}=\left(a_{t}\right) \in A^{-\infty}\right\}$

2) For every $s$, there exists $M(s)$ such that for every $c \hat{c}(s)$, $\left\|c_{t}\right\|<\frac{M(s)}{\lambda^{* t}}$

3) For all s in $R_{+}^{n}, \hat{C}(s)$ is convex and compact in the product topology of $\left(R^{n}\right)^{\infty}$.

Proof : The proof of 1) may be found in Dana et al. [1987] lemma 4.4. To prove 2, let $\underset{\sim}{c} \in \hat{C}(s)$, then by 1$)$; for some $\underset{\sim}{a}, c_{0}+\underset{i}{i}\left(\prod_{\tau=1}^{t} a_{T}\right) c_{t} \leqslant s$. Let $p *$ be as in proposition 2.1 .

$$
\text { Then } p * c_{0}+\sum_{1}^{\infty} \lambda^{t} p * c_{t}<p * c_{0}+\sum_{1}^{\infty} p *\left(\prod_{t=1}^{t} a_{\tau}\right) c_{t}<p * . s
$$


Therefore $p * c_{t} \leqslant \frac{p * \cdot s}{\lambda^{* t}}$.

As $\hat{C}(s)$ is trivially closed for the product topology, the proof of 3 ) follows easily from 1 ) and 2 ).

1

For further use let us introduce the following cone

$F_{+}=\left\{\underset{\sim}{x} \varepsilon\left(R_{+}^{n}\right)^{\infty} \mid x_{0}+\sum_{t=1}^{\infty}\left(\prod_{\tau=1}^{t} a_{\tau}\right) x_{t}<\infty, \underset{\sim}{a}=\left(a_{t}\right) \in A^{\infty}\right\}$

It follows from proposition 2.2 that $F_{+}=U \hat{C}(s)$. We also have:

$$
s \varepsilon R_{+}^{n}
$$

Proposition 2.3 - Under assumptions $P_{1}-P_{3}, C_{1}-C_{5}$

For every $i, U^{i}$ is well defined on $F_{+}$and is continuous on $\hat{C}(s)$ endowed with the product topology.

Proof - From proposition 2.2. there exists $M(s)>0$ such that

$\left\|C_{t}\right\| \leqslant \frac{M(s)}{(\lambda *)^{t}}$. From $C_{4}$ and $C_{5}$, one has :

$$
\sum_{t=0}^{\infty}\left(\delta^{i}\right)^{t} u^{i}\left(c_{t}\right)<M(s)^{\mu_{i}} M_{i} \sum_{t=0}^{\infty}\left(\frac{\delta^{i}}{\lambda_{\lambda} \mu_{i}}\right)^{t}<+\infty
$$

Hence $\sum_{t=T}^{\infty}\left(\delta^{i}\right)^{t} u^{i}(c)$ converges uniformly to $U^{i}(c) ; U^{i}$ is therefore cont inuous. 


\section{Existence of compet it ive equilibria}

\section{Competitive equilibrium paths}

We define an equilibrium path $\underset{\sim}{\bar{x}}, \underset{\sim}{\bar{y}}, \bar{a}, \bar{p})$ by the following conditions:

(3) (utility maximization) $-\forall i, \underset{\sim}{x^{i}} \operatorname{maximizes} U^{i}\left({\underset{\sim}{x}}^{i}\right)$ subject to $\underset{\sim}{x^{i}} \varepsilon\left(R_{+}^{n}\right)^{\infty}$ and $\bar{p} \cdot x^{i} \leq \bar{p}_{0} \cdot s^{i}$.

(4) (profit maximization) $-\forall j, \underset{\sim}{\sim}{ }^{j} \varepsilon\left(A^{j}\right)^{\infty}, \underset{\sim}{\sim y^{j}} \varepsilon\left(R_{+}\right)^{\infty}$,

$$
\sum_{t=1}^{\infty}\left(\bar{p}_{t}^{j}-\bar{p}_{t-1} \cdot a_{t}^{j}\right) y_{t}^{j} \leqq \sum_{t=1}^{\infty}\left(\bar{p}_{t}^{j}-\bar{p}_{t-1} \cdot \bar{a}_{t}^{j}\right) \bar{y}_{t}^{j}=0
$$

(5) (market clearing) - $(\underset{\sim}{\bar{x}}, \underset{\sim}{\bar{y}}, \bar{a})$ is an attainable allocation path with $\leqq$ replaced by $=$ in relations (1) and (2).

Equilibrium price paths $\underset{\sim}{\bar{p}}=\left(\bar{p}_{t}\right)$ must belong to the cone $\left\{p_{\varepsilon} \varepsilon\left(R_{+}^{n}\right)^{\infty} / p_{t}^{j} \leqq \inf _{a_{\varepsilon} A^{j}} p_{t-1} \cdot a^{j}, \forall j=1, \ldots, n\right\}$. In the following, we will concentrate on equilibrium price paths which allow every producer to get at each period a maximum profit (equal to 0 ) whatever may be the level $y_{t}^{j}$ of production. Such prices satisfy the induction relation :

$$
-\forall t \geqq 1, \forall j=1, \ldots, n, \bar{p}_{t}^{j}=\min _{a_{\varepsilon A}^{j}} \bar{p}_{t-1} \cdot a^{j}
$$

Let us denote by $f: R_{+}^{n}+R_{+}^{n}$ the function defined by $: f_{j}(p)=\inf _{j} p \cdot a^{j}$. $a^{j} \in A^{j}$ If $p \gg 0, f_{j}(p)$ equals the minimum cost to produce one unit of commodity $j$.

\section{Proposition 3.1}

Under assumptions $P_{1}-P_{3}$ and $C_{1}-C_{5}$ let $(\underset{\sim}{\bar{x}}, \bar{\sim}, \underset{\sim}{\bar{y}}, \bar{\sim}, \bar{p})$ be a competitive equilibrium path of $\mathcal{E}$. If we assume $\bar{p}_{0} \gg 0$ and if we define 
$\overline{\bar{p}}=\left(\overline{\bar{p}}_{t}\right)$ by $\overline{\bar{p}}_{0}=\bar{p}_{0}$ and $\overline{\bar{p}}_{t}=f^{t}\left(\bar{p}_{0}\right) \forall t \geqq 1$, then $\left(\bar{x}_{\sim}, \underset{\sim}{\bar{y}}, \bar{\sim}, \bar{\sim}, \bar{\sim}\right)$ is a competitive equilibrium path of $\mathcal{E}$ :

Therefore every competitive equilibrium can be replaced by another competitive equilibrium with same quantities but which prices satisfy the relation $p_{t}=f^{t}\left(\bar{p}_{0}\right)$.

\section{proposition 3.2}

Under assumptions $P_{1}-P_{3}$ and $C_{1}-C_{5}, \mathcal{C}$ admits an equilibrium $\left.\underset{\sim}{\bar{x}}, \underset{\sim}{\bar{y}}, \underset{\sim}{\bar{a}}, \bar{\sim}\right)$ with $\bar{p}_{t}=f t\left(\bar{p}_{0}\right), \bar{p}_{0} \gg 0$ and

$$
\bar{y}_{t}=\bar{c}_{t}+\sum_{k=t+1}^{\infty}\left(\prod_{t=t+1}^{k} \bar{a}_{\tau}\right) \bar{c}_{k} .
$$

The proofs of proposition 3.1 and 3.2 may be found in Dana et a). [1987]. Following Peleg and Ryder [1972], in order to study the asymptotic properties of equilibria we shall characterize them by conditions decentralized in time.

\section{Characterization of compet itive equilibria}

Proposition $3.3-$ Assume $P_{1}-P_{3}, C_{1}-C_{5}$

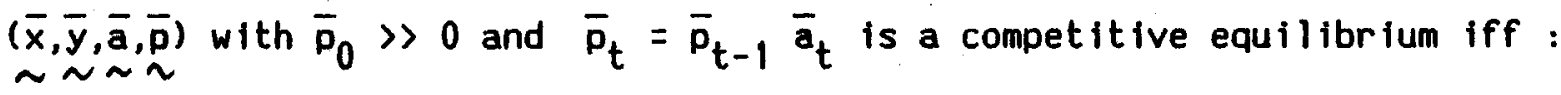

(i) For every $i$ such that $s^{i}>0$, there exists $d^{i}>0$ such that $\forall t, \forall c \in R_{+}^{n}$ :

$$
\left(\delta^{i}\right)^{t} u^{i}\left(\bar{x}_{t}^{i}\right)-d^{i} \bar{p}_{t} \bar{x}_{t}^{i} \geqslant\left(\delta^{i}\right)^{t} u^{i}(c)-d^{i} \bar{p}_{t} c
$$

$$
\bar{c}_{0}+\sum_{1}^{\infty}\left(\prod_{\tau=1}^{t} \bar{a}_{\tau}\right) \bar{c}_{t}=s ; \bar{y}_{t}=\bar{c}_{t}+\sum_{k=t+1}^{\infty}\left(\sum_{\tau=t+1}^{k} \bar{a}_{\tau}\right) \bar{c}_{k}
$$

(iii) $\underset{\sim}{\bar{p}} \cdot \bar{x}_{\sim}^{i}=\bar{p}_{0} \cdot s^{i}, \forall i$ 


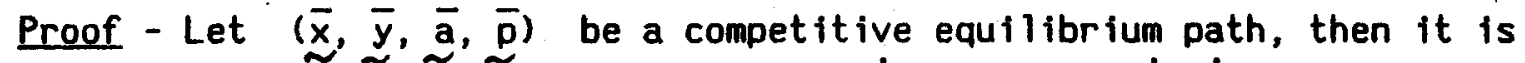
optimal and $(i i)$ is satisfied. Moreover $\underset{\sim}{-i}$ maximizes $U^{i}\left(x_{\sim}^{i}\right)$ under the constraint $\underset{\sim}{x^{i}} \in F_{+}, \bar{p} \underset{\sim}{x_{\sim}^{i}}<\bar{p}_{0} s^{i}$. The functions $U^{i}\left(\underset{\sim}{x^{i}}\right)$ and $\bar{p} \underset{\sim}{x^{i}}$ are well defined on $\tilde{F}_{+}$. Since $S^{i}>0$ and $p_{0} \gg 0$ the Slater condition is satisfied and therefore we can use theorem 5.3.1.2 of Hurwicz [1958]. Thus for every $i$ there exists $d^{i} \in R_{++}$such that :

$$
U^{i}\left(\bar{x}^{i}\right)-d^{i} \bar{p} \underset{\sim}{\bar{x}^{i}} \geqslant U^{i}(\underset{\sim}{c})-d^{i} \underset{\sim}{\bar{p}} \cdot \underset{\sim}{c}, \underset{\sim}{\forall c \in F}+
$$

Let us consider the sequence $c_{\tau}=\bar{x}_{\tau}$ if $\tau \neq t$ and $c_{t}=c$. Clearly $\underset{\sim}{c} \in F_{+}$and we get (7).

Conversely assume ( $i),(i i)$ and (iii). Then summing up inequalities we have :

$$
\begin{aligned}
\sum_{t=0}^{T}\left(\delta^{i}\right)^{t} u^{i}\left(\bar{x}_{t}\right)-d^{i} \sum_{t=0}^{T} \bar{p}_{t} \cdot \bar{x}_{t} \\
\\
\qquad \sum_{t=0}^{T}\left(\delta^{i}\right)^{t} u^{i} \underbrace{(c)}_{t}-d^{i} \sum_{t=0}^{T} \bar{p}_{t} c_{t}^{c}, \forall c_{t} \in R_{t}^{n}
\end{aligned}
$$

As $\underset{\sim}{\bar{c}}$ fulfills (ii), $\bar{c} \in F_{+}$, and therefore all $\underset{\sim}{\bar{x}^{i}} \in F_{+}$. Let $\underset{\sim}{c^{i}} \in\left(R_{+}^{n}\right)^{\infty}$ be such that $\bar{p} \cdot \underset{\sim}{c}<\bar{p}_{0} s^{i}$, then $c^{i} \in F_{+}$. Let $T \rightarrow \infty$, then all the infinite sums are well defined. We thus have $(8)$. Using $(i i i)$ we obtain $U^{i}\left(\bar{c}^{i}\right) \geqslant U^{i}\left(c^{i}\right)$. Therefore $(\bar{x}, \bar{y}, \bar{a}, \bar{p})$ is a competitive equilibrium. 


\section{Asymptotic properties of equilibrium prices}

4.1. Convergence properties of equilibrium paths

Let $f: R_{+}^{n}+R_{+}^{n}$ be defined by $f_{j}(p)=\inf p_{j_{f}} a^{j}$. For further use let us introduce the following functions.

Let $p, q \in R_{+}^{n}$ be strictly positive. We denote :

$$
\begin{aligned}
& M(p, q)=\inf \{\lambda / p \leqq \lambda a\}=\max _{k} \frac{p_{k}}{q_{k}} \\
& m(p, q)=\sup \{\lambda / p \geqq \lambda a\}=\min _{k} \frac{p_{k}}{q_{k}}
\end{aligned}
$$

Then, if $\lambda>0$ we have for all $p$ and $q \gg 0$ :

$$
M(\lambda p, q)=\lambda M(p, q) ; m(\lambda p, q)=\lambda m(p, q) ; m(p, q) q \leqq p \leqq M(p, q) q .
$$

Let us recall that $f$ is an increasing, homogeneous of degree 1 continuous mapping and

$$
m(p, q)<m(f(p), f(q))<M(f(p), f(q))<M(p, q)
$$

and that under $P_{1}-P_{3} f$ has a unique strictly positive eigenvector $p *$ such that $f(p *)=\lambda * p *=p *$ a* ( $\lambda *$ and $p *$ are those defined in proposition 2.1$)$. Let us prove the following result.

Proposition 4.1 - Assume $P_{1}-P_{3}$ then for every $P_{0} \gg 0$, the sequence $\frac{f^{t}\left(p_{0}\right)}{\left\|f^{t}\left(p_{0}\right)\right\|}$ converges to $\frac{p *}{\|p *\|}$ and $\frac{f^{t}\left(p_{0}\right)}{\lambda *^{t}}$ converges to $\mu p *$ for some $\mu>0$.

Proof - The proof of the first statement may be found in Dana et al. [1987]. We prove now the second statement. 
It follows from $f\left(p_{0}\right) \leqslant p_{0}$ a* that :

$$
\begin{aligned}
& f^{t}\left(p_{0}\right)<p_{0}(a *)^{t} \leqslant M\left(p_{0}, p *\right) p *(a *)^{t}=(\lambda *)^{t} M\left(p_{0}, p *\right) p * \\
& f^{t}\left(p_{0}\right) \leqslant f^{t}(p *) m\left(p_{0}, p *\right)=(\lambda *)^{t} m\left(p_{0}, p *\right) p * .
\end{aligned}
$$

Therefore $\frac{f^{t}\left(p_{0}\right)}{\lambda^{\star t}}$ is bounded above and below. Let $p_{k}$ be a cluster point of $\frac{f^{t}\left(p_{0}\right)}{\lambda^{* t}}$. As $\frac{f^{t}\left(p_{0}\right)}{\left\|f^{t}\left(p_{0}\right)\right\|}+\frac{p *}{\|p *\|}, p_{k}=\alpha_{k} p *$ for some $\alpha_{k}>0$.

As $\quad M\left(f^{t}\left(p_{0}\right), \lambda * p *\right)=M\left(\frac{f^{t}\left(p_{0}\right)}{\lambda x^{t}}, p *\right)<M\left(f^{t-1}\left(p_{0}\right), \lambda x^{t-1} p *\right)=M\left(\frac{f^{t-1}\left(p_{0}\right)}{\left.\lambda x^{t-1}, p *\right)}\right.$ the sequence $M\left(\frac{f^{t}\left(p_{0}\right)}{\lambda *^{t}}, p *\right)$ is therefore non increasing. Let $\mu=\inf _{t} M\left(\frac{f^{t}\left(p_{0}\right)}{\lambda^{t}}, p *\right)$. As $M$ is continuous we have $: \mu=M\left(\alpha_{k} p *, p *\right)=\alpha_{k}$. Therefore the sequence $\frac{f^{t}\left(p_{0}\right)}{\lambda *^{t}}$ has a unique cluster point $\mu p^{*}$ and is therefore convergent. 
Proposition 4.2 - Assume $P_{1}-P_{4}$. Let $\underset{\sim}{(\bar{x}, \bar{\sim}, \underset{\sim}{\bar{a}}, \bar{\sim}, \bar{p})}$ be a competitive equilibrium with $\bar{p}_{0} \gg 0$ and $\bar{p}_{t}=\bar{p}_{t-1} \bar{a}_{t}$ for every $t$. then $\bar{a}_{t}+a x$.

Proof Let $\bar{p}_{t}=\bar{p}_{t-1} \bar{a}_{t}$. Then $\lambda * \frac{\bar{p}_{t}}{(\lambda *)^{t}}=\frac{\bar{p}_{t-1}}{\left(\lambda_{*}\right)^{t-1}} \bar{a}_{t}$. There exists $\mu_{1}>0$ and $\mu_{2}>0$ such that for $t$ large enough

$0 \ll \mu_{1} p * \ll \frac{\bar{p}_{t}}{(\lambda *)^{t}}<\mu_{2} p *$. Therefore $\lambda * \mu_{2} p * \geqslant \mu_{1} p * \bar{a}_{t}$.

The sequence $\underset{\sim}{\bar{a}}$ is therefore uniformmy bounded.

Let $\overline{\bar{a}}$ be a cluster point of $\underset{\sim}{\bar{a}}$. Since $\lambda * \frac{\bar{p}_{t}}{\left(\lambda_{*}\right)^{t}}=\frac{\bar{p}_{t-1}}{(\lambda *)^{t-1}} \bar{a}_{t}$

and $\frac{\bar{p}_{t}}{(\lambda *)^{t}} \underset{t \rightarrow \mu}{\rightarrow \mu}$, $\overline{\bar{a}}$ satisfies $\lambda * p^{*}=p * \overline{\bar{a}}$. Under $P_{4}, \overline{\bar{a}}=a *$.

4.2. Asymptotic properties of the equilibrium consumption and production path

We have the following proposition.

\section{Proposition 4.3}

$$
\begin{aligned}
& \text { Assume } P_{1}-P_{4}, C_{1}-C_{5} . \\
& \text { Define } g_{i}=\left(\frac{\delta^{i}}{\lambda x}\right) \frac{1}{1-\mu_{i}} \text { for } i=1, \ldots, m \\
& \text { and } g=\max _{i} g_{i}
\end{aligned}
$$


15

Let $(\underset{\sim}{\bar{x}}, \underset{\sim}{\bar{y}}, \bar{\sim}, \bar{\sim})$ be an equilibrium path with $\bar{p}_{t}=f^{t}\left(\bar{p}_{0}\right), \forall t$.

Then there exist $A_{i}>0, B_{i}>0, A>0, B>0$ such that :

1) $\forall i, B_{i}<\inf _{t} \frac{\left\|\bar{x}_{t}^{-i}\right\|}{g_{i}^{t}}<\sup _{t} \frac{\left\|\bar{x}_{t}^{-i}\right\|}{g_{i}^{t}}<A i$

2) $B<\inf _{t} \frac{\left\|\bar{y}_{t}\right\|}{g^{t}}<\sup _{t} \frac{\left\|\bar{y}_{t}\right\|}{g^{t}}<A$

Proof

1 - By proposition 3.3, there exists $d^{i}>0$ such that

$$
\left(\frac{\delta}{\lambda x}\right)^{i} u^{i}\left(\bar{x}_{t}^{i}\right)-d^{i} \frac{f^{t}\left(\bar{p}_{0}\right)}{(\lambda x)^{t}} \bar{x}_{t}^{i} \geqslant\left(\frac{\delta^{i}}{\lambda x}\right)^{t} u^{i}(c)-d^{i} \frac{f^{t}\left(\bar{p}_{0}\right)}{(\lambda x)^{t}}, c, \forall c \in R_{+}^{n}
$$

Define $\overline{\bar{x}}_{t}^{i}=\bar{x}_{t}^{i}\left(g^{i}\right)^{-t}$. By proposition 4.1 , for $t$ sufficiently large

$\mu_{1} p * \geqslant \frac{f^{t}\left(\bar{p}_{0}\right)}{(\lambda *)^{t}}>\mu_{2} p * \gg 0$, for some $\mu_{1}>0, \mu_{2}>0$

Under $C_{4}$, one has, for $t$ large enough :

$M_{i}\left\|\overline{\bar{x}}_{t}^{i}\right\|^{\mu_{i}}-d^{i} \mu_{2} p * \overline{\bar{x}}_{t}^{i} \geqslant m_{i}\left\|c^{\prime}\right\| \|^{\mu_{1}}-d^{i} \mu_{1} p * c^{\prime}, \forall c^{\prime} \in R_{+}^{n}$

In other words

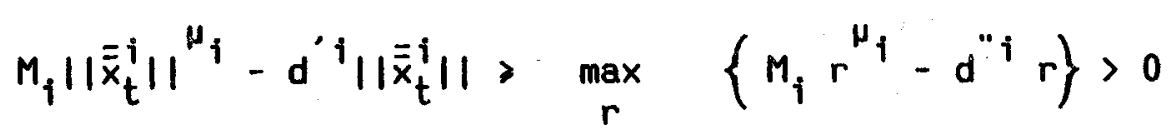

where $d^{i}=d^{i} \mu_{2} \min _{j} p_{j}^{*}$ and $d^{\prime \prime}=d^{i} \mu_{1} \max _{j} p_{j}^{*}$. 
Therefore $\inf _{t}\left\|\overline{\bar{x}}_{t}^{i}\right\|=B_{i}>0$ and $\sup _{t}\left\|\overline{\bar{x}}_{t}^{i}\right\|<A_{i}$ with $A_{i}=\left(\frac{M_{i}}{d^{\prime i}}\right) \frac{1}{1-\mu_{i}}$

2. By proposition 3.2 , one has :

$$
\bar{y}_{t}=\bar{c}_{t}+\sum_{k=t+1}^{\infty}\left(\sum_{\tau=t+1}^{k} \bar{a}_{\tau}\right) \bar{c}_{k}
$$

where $\bar{c}_{k}=\sum_{i=1}^{m} \bar{x}_{k}^{i}$ for $k \geqslant t$

Let $I=\left\{i \mid g^{i}=g\right\}$

For $t$ sufficiently large

$$
\frac{\bar{y}_{t}}{g^{t}} \geqslant \frac{i \bar{x}_{t}^{i}}{g^{t}} \geqslant \frac{\sum_{i \in I}^{[} \bar{x}_{t}^{i}}{g^{t}}+\varepsilon \geqslant \underset{i \in I}{\left[B_{i}+\varepsilon\right.}
$$

On the other hand, from $C_{5}$, one has $g \lambda x<1$

Let $\varepsilon^{\prime}$ be such that $g \lambda *\left(1+\varepsilon^{\prime}\right)<1$. For $t$ large enough, since $\bar{a}_{t}$ converges to ax:

$$
\frac{\bar{y}_{t}}{g}<\frac{\bar{c}_{t}}{g^{t}}+\sum_{k=t+1}^{\infty}\left(g\left(1+\varepsilon^{\prime}\right) a x\right)^{k-t} \frac{\bar{c}_{k}}{g^{k}}
$$

Let $e=(1,1, \ldots, 1)$

Then $\frac{\bar{y}_{t}}{g} \leqslant \sup _{t} \frac{\left\|\bar{c}_{t}\right\|}{g^{t}}\left(I-g\left(\mid+\varepsilon^{\prime}\right) a x\right)^{-1} e$ 


\section{Corollary 4.1}

The equilibrium consumption and production paths tend to inf inity if $\delta^{i}>\lambda *$, to zero if $\delta^{i}<\lambda *$ and remain bounded be low and above by strictly positive vectors if $\delta^{i}=\lambda *$.

\subsection{A particular case: the case of homogeneous utilities}

In this part, we assume :

$c_{4}^{\prime}: \forall i$, there exists $\left.\mu^{i} \in\right] 0,1\left[\right.$ such that $\forall x \in R_{+}^{n}, \forall \lambda \geqslant 0, u^{i}(\lambda x)=\lambda^{\mu} u(x)$

\section{$\underline{\text { Remark }}$}

Since $u^{i}(x)=u^{i}\left(\|x\| \frac{x}{\|x\|^{\prime}}\right)$, one has $m_{i}\|x\|^{\mu_{i}} \leqslant u^{i}(x) \leqslant\|x\|^{\mu_{i}} M_{i}$, with $m_{i}=\min _{\|x\|=1} u^{i}(x)$ and $M_{i}=\max _{\|x\|} u^{i}(x)$

Hence $C_{4}^{\prime}$ implies $C_{4}$.

We next prove that under $c_{4}^{\prime}$ every competitive equilibrium converges to a competitive equilibrium with constant growth rate that we define next.

\section{Definition}

$(\hat{x}, \hat{\sim}, \hat{\sim}, \hat{\sim}, \hat{p})$ is competitive equilibrium with constant growth rate $g$ if it is a competitive equilibrium assoclated with some initial endowments and if there exists $g>0$ such that $\hat{x}_{t}^{i}=g^{t} x_{0}^{i}$ for every $i$ and $\hat{y}_{t}=g^{t} y_{0}$.

\section{Proposition 4.4}

Assume $C_{1}-C_{4}^{\prime}, C_{5}, P_{1}-P_{4}$.

Let $g$ be defined as in proposition 4.3 . 
18

and $(\bar{x}, \bar{\sim}, \bar{\alpha}, \bar{p})$ be a competitive equilibrium with $\bar{p}_{t}=f^{t}\left(\bar{p}_{0}\right), \forall t$. There exists a competitive equilibrium $(\underset{\sim}{\hat{x}}, \hat{\sim}, \hat{a}, \hat{\alpha}, \hat{p})$ with constant growth rate $g$ such that one has :

$$
\begin{aligned}
& \hat{a}_{t}=a *, \forall t, \text { and } \hat{x}_{t}^{i}=g^{t} \hat{x}_{0}^{i}, \forall t, \text { such that } \forall i=1, \ldots, m \frac{\bar{x}_{t}}{g^{t}}+\hat{x}_{0}^{i} . \\
& \forall i=1, \ldots, n \frac{\bar{y}_{t, i}}{\hat{y}_{t, i}}+1, \bar{a}_{t} \rightarrow \text { a* and } \frac{\bar{p}_{t}^{j}}{\hat{p}_{t}^{j}}+1, \forall j=1, \ldots n .
\end{aligned}
$$

Proof

Let $x_{0}^{i}$ be a cluster point of $\left\{\frac{\bar{x}_{t}^{i}}{g^{t}}\right\}$.

By proposition 3.3 , there exists $d^{1}>0$ such that

$$
\left(\frac{\delta^{i}}{\lambda x}\right) u^{i}\left(\bar{x}_{t}^{i}\right)-d^{i} \frac{f^{t}\left(\bar{p}_{0}\right)}{(\lambda x)^{t}} \bar{x}_{t}^{i} \geqslant\left(\frac{\delta^{i}}{\lambda x}\right)^{t} u^{i}(c)-d^{i} \frac{f^{t}\left(\bar{p}_{0}\right)}{(\lambda x)^{t}} c, \forall c \in R_{+}^{m} .
$$

Since $u^{i}$ is homogeneous and $\frac{f^{t}\left(\bar{p}_{0}\right)}{(\lambda *)^{t}}+\mu p *$, one has :

$$
u^{i}\left(x_{0}^{i}\right)-d^{i} \mu p * \cdot x_{0}^{i} \geqslant u^{i}(c)-d^{i} \mu p * c, \forall c \in R_{+}^{n}
$$

Since $u^{i}$ is strictly concave, such a $x_{0}^{i}$ is unique. Hence $\frac{\bar{x}_{t}}{g_{t}^{i}}$ converges to $x_{0}^{1}$.

Let $I=\left\{i \mid g^{i}=g\right\}$ Define $\hat{x}_{0}^{i}=0$ for $i \notin I$ and $\hat{x}_{0}^{i}=x_{0}^{i}$ for $i \in I$. Obviously $\frac{\bar{x}_{t}^{i}}{g^{t}}+\hat{x}_{0}^{i}$ 
Consider now the feasibility relation :

$\frac{i \bar{x}_{t}^{i}}{g^{t}}+g \bar{a}_{t} \frac{\bar{y}_{t+1}}{g^{t+1}}=\frac{\bar{y}_{t}}{g^{t}}$

Let $\gamma_{0}$ be a cluster point of $\left\{\frac{\bar{y} t}{g t}\right\}, \gamma_{1}$ be one of $\left\{\frac{\bar{y}_{t+1}}{g}\right\}, \ldots$

$\gamma_{k}$ be one of $\left\{\frac{\bar{y} t+k}{g^{t+k}}\right\}$.

Let $\hat{c}_{0}=\sum_{i} \hat{x}_{0}^{i}$. Since $\bar{a}_{t} \rightarrow$ a* (proposition 4.2) then

$\hat{c}_{0}+g$ ax $\gamma_{1}=\gamma_{0}$

$\hat{c}_{0}+g a * \gamma^{K}=\gamma^{k-1}$

Hence $\gamma^{t}-\gamma^{t+1}=g a *\left(\gamma^{t+1}-\gamma^{t+2}\right)$ for $0<t<k-2$ and
$\gamma^{0}-\gamma^{1}=(g a x)^{k-1}\left(\gamma^{k-1}-\gamma^{k}\right)$

Since $\gamma^{t} \leqslant A^{\prime}, \forall t$, (proposition 4.3)

$\left\|\gamma^{0}-\gamma^{1}\right\|<2 \|(g \text { ax })^{k-1} A^{\prime} \| \rightarrow 0$. Therefore

$r^{0}=\gamma^{1}=(I-g a x)^{-1} \hat{c}$ and . The sequence $\left\{\frac{\bar{y}_{t}}{t_{g}}\right\}$ converges to $(I-g a x)^{-1} \hat{c}_{0}$

Define :

$\hat{s}^{i}=\sum_{t=0}^{\infty}(g a x) t \hat{x}_{0}^{i}$ 
By applying proposition 3.3 , one can see that $(\hat{x}, \hat{y}, \hat{a}, \hat{\sim})$ defined as follows :

$$
\begin{aligned}
& \hat{x}_{t}^{i}=g^{t} \hat{x}_{0}^{i}, \forall i, \forall t \\
& \hat{y}_{t}=g^{t}(I-g a *)^{-1} \hat{c}_{0}, \forall t \\
& a_{t}=a *, \forall t \\
& \hat{p}_{t}=\mu p *(\lambda *)^{t}, \forall t
\end{aligned}
$$

is a competitive equilibrium with $\hat{\mathbf{s}}^{i}$ as initial endowments.

\section{Remarks :}

1. Using proposition 3.3 one can prove that under $P_{1}-P_{4}, C_{1}-C_{3}$ and

$$
c_{5}^{\prime}: \max _{i} \delta^{i}<\min (\lambda *, 1)
$$

there exists a competitive equilibrium $(\bar{x}, \bar{y}, \bar{a}, \bar{p})$ such that:

$$
\bar{a}_{t} \rightarrow a *, \frac{\bar{p}_{t}}{(\lambda *)^{t}} \rightarrow \mu p^{\prime}, \bar{x}_{t} \rightarrow 0, \bar{y}_{t} \rightarrow 0
$$

2. One can also prove that, if $C_{4}$ and $C_{5}$ are replaced by

$$
\begin{aligned}
& C_{4}^{\prime \prime}: u^{i}(x)<M_{i}\|x\|, \forall x \in R_{+}^{n}, \forall i \\
& c_{5}^{\prime}: \max \delta^{i}<\min (\lambda *, 1)
\end{aligned}
$$


Then there exists $t_{0}$ such that

$\forall t>t_{0}, \bar{x}_{t}^{i}=0, \forall i, \bar{y}_{t}=0$

Where $\underset{\sim}{\bar{x}}, \bar{\sim}$ are equilibrium consumption and production paths.

Indeed, under these assumptions, proposition 3.3 still holds, and in particular, there exists $d^{i}>0$ such that relation $(7)$ is verified. Since $\frac{\bar{p}_{t}}{\left(\lambda_{*}\right)}$ converges to $\mu p * \gg 0$ when $t \rightarrow \infty$, one has, for $t$ large enough :

$\forall c \in R_{+}^{n},\{0\},\left(\frac{\delta^{i}}{\lambda^{*}}\right)^{t} \frac{u^{i}(c)}{\|c\|}-d^{i} \frac{\bar{p}_{t}}{\left(\lambda_{*}\right)^{t}} \frac{c}{\|c\|}<M^{i}\left(\frac{\delta^{i}}{\lambda^{*}}\right)^{t}-d^{i} \frac{\bar{p}_{t} \cdot c}{\left(\lambda_{*}\right)^{t}|| c||}<0$.

Hence, for $t$ large enough, relation (7) is verified iff $\bar{x}_{t}^{i}=0$; from proposition 3.2 one has $\bar{y}_{t}=0$. 


\section{REFERENCES}

Bewley, T. (1982) - "An Integration of Equilibrium Theory and Turnpike Theory", Journal of Economic Theory, 10, 233-267.

Coles, J. (1985) - "Equilibrium Turnpike Theory with Constant Returns to Scale and possibly Heterogenous Discount Factors", International Economic Review, 26, ,671-679.

Coles, J. (1986) - "Equilibrium Turnpike Theory with Time-Separable Utility", Journal of Economic Dynamics and Control, 10, 367-394.

Dana, R.A, Florenzano M., Le Van C. and Lévy D. (1987) - "Production Prices and General Equilibrium Prices : a Long-Run Property of a Leontief Economy with an Unlimited Supply of Labour", Working Paper CEPREMAP, $n^{\circ} 8734$.

Hurwicz, L. (1958) - "Programming in Linear Spaces", in Studies in Linear and Non Linear Programming, Arrow-Hurwicz-Uzawa Ed, Stanford University Press, Stanford.

Mckenzie, L.W. (1963) - "Turnpike Theorem of Morishima", Review of Economic Studies, $30, n^{\bullet} 3,169-1976$.

Morishima, M. (1961) - "Proof of Turnplke Theorem: The No Joint Production Case", Review of Economic Studies, $28, n^{\bullet} 76,89-97$.

Peleg, B. and Ryder H. (1972) - "On Optimal Consumption Plans in a Multisector Economy", Review of Economic Studies, 39, 159-169.

Samuelson, P. and Solow R. (1953) - "Balanced Growth under Constant Returns to Scale", Econometrica, $21, n^{\bullet} 3,412-424$.

Yano, M. (1984) - "Competitive Equilibria on Turnpikes in a Mc Kenzie Economy. I. A Neighbourhood Turnpike Theorem", International Economic Review, 25, $n \cdot 3,695-717$.

Yano, M. (1985) - "Competitive Equilibria on Turnpikes in a Mc Kenzie Economy. II. An Asymptotic Turnpike Theorem", International Economic Review, $26, n^{\bullet} 23$, $661-669$. 